Meta

Journal des traducteurs

Translators' Journal

\title{
Une rétrospective et un regard sur l'avenir
}

\section{J. Albert Bachrach}

Volume 32, numéro 2, juin 1987

Vers l'an 2000. La terminotique, bilan et prospectives

Objectives: Year 2000 Terminotics. State of the Art, Prospects for the Future

URI : https://id.erudit.org/iderudit/001938ar

DOI : https://doi.org/10.7202/001938ar

Aller au sommaire du numéro

Éditeur(s)

Les Presses de l'Université de Montréal

ISSN

0026-0452 (imprimé)

1492-1421 (numérique)

Découvrir la revue

Citer ce document

Bachrach, J. A. (1987). Une rétrospective et un regard sur l'avenir. Meta, 32(2),

97-101. https://doi.org/10.7202/001938ar d'utilisation que vous pouvez consulter en ligne.

https://apropos.erudit.org/fr/usagers/politique-dutilisation/ 


\title{
UNE RÉTROSPECTIVE ET UN REGARD SUR L'AVENIR
}

\author{
J. Albert BACHRACH \\ Directeur honoraire, Ancien directeur-coordinateur, \\ Service de traduction à moyen et à long termes, \\ Commission des communautés européennes, Luxembourg, Grand-Duché
}

La rédaction de $M E T A$ a décidé de consacrer un de ses numéros à la situation de la terminotique (terminologie et informatique) vers l'an 2000. C'est en pensant à ce début du $21^{\mathrm{e}}$ siècle que l'on est tenté de faire de la prospective. Cette science a depuis la fin de la Deuxième Guerre mondiale résulté en tant de prédictions et d'espérances que la réalité souvent bien moins rose, a dû réveiller cruellement tous ceux qui avaient cru fermement à une haute conjoncture durable et sans fin. Aussi n'avons-nous pas osé prendre le risque de faire des prévisions hasardeuses.

Étant donné que le présent ne peut être que le résultat du passé et que le futur repose invariablement sur ce présent si éphémère, il ne sera pas inutile de jeter un regard en arrière. Ceci est d'autant plus nécessaire si l'on désire éviter qu'à l'avenir, dans le domaine de la terminotique comme dans tant d'autres, l'Amérique soit redécouverte pour la nième fois !

Ce danger est très réel, car à l'époque de l'informatique, les innombrables changements, les progrès se suivent à une allure vertigineuse. Il est alors quasiment impossible de rester à jour même à l'aide d'outils de recherche très sophistiqués. Dans le passé, beaucoup de doubles emplois auraient pu être évités si tous les créateurs de bases de données terminologiques avaient étudié de façon plus approfondie les réalisations déjà existantes. Aussi l'incompatibilité, dont souffrent encore aujourd'hui les clients des banques de terminologie, aurait pu être empêchée.

Lorsque à la fin des années 50 et au début des années 60 les premiers efforts timides de création de dictionnaires électroniques ont été tentés, leurs initiateurs furent souvent inspirés par les essais balbutiants en traduction automatique. Ils perdirent de vue l'objectif à atteindre. Il ne s'agit point de créer de quelconques stocks comprenant pêlemêle des mots de tout genre assortis d'équivalents dans d'autres langues parmi lesquels l'ordinateur devait choisir dans le but de sortir une traduction, qui dans le meilleur des cas n'était qu'une reproduction mot à mot du texte original dans la langue d'arrivée.

Le dictionnaire électronique était en premier lieu destiné à servir d'outil de travail au traducteur humain. Or, rares furent les chercheurs qui se donnèrent la peine de considérer de près le déroulement du processus de la traduction. Longtemps on a imaginé que l'on traduisait des mots isolés, or tel n'est point le cas. Le traducteur traduit un groupe de mots qui ensemble représentent une notion précise, justement parce que ces mots sont groupés dans un certain ordre et constituent ainsi une phrase ou parfois un segment de phrase, qui à lui seul a une signification déterminée. C'est pour cette raison que les grands dictionnaires de langues contiennent pour chaque mot un ou plusieurs exemples d'emploi, qui illustrent les différentes significations. Malgré le grand nombre de néologismes qui sont continuellement introduits aussi bien dans la langue courante que dans la langue de spécialité, les cas restent nombreux où le même mot, le même terme est employé avec des significations très différentes. 
Les dictionnaires automatiques ou banques de terminologie, tout comme les dictionnaires en général, ne sont point destinés uniquement aux traducteurs, mais bien sûr également aux rédacteurs et à tous ceux qui se servent des langues comme moyen de communication. Mais il est évident que ce sont avant tout les traducteurs des grandes organisations tant nationales qu'internationales ainsi que ceux des institutions des Communautés européennes qui dès le prime abord ont été visés comme principaux clients des banques de terminologie. Dans le cas présent il s'agissait des traducteurs au service de la Haute Autorité de la CECA (Communauté européenne du charbon et de l'acier). Ceci étant, î fallait, avant de commencer à créer des systèmes de stockage en mémoire de termes et des méthodes de consultation de ces stocks, d'abord se rendre compte des problèmes auxquels ils ont à faire face dans l'exercice de leur fonction.

Les chercheurs des années 50 et 60 semblent avoir été médusés par les possibilités offertes par la traduction automatique. Ils croyaient que la machine écarterait l'homme dans un très proche avenir. Or l'expérience a démontré que trente années plus tard la plupart des traductions sont toujours effectuées par des traducteurs humains. Et c'est précisément eux qui ont besoin d'outils informatisés, tels que les banques de terminologie et/ou les machines de traitement de texte.

Car, quels étaient et sont les problèmes auxquels le traducteur devait et doit toujours trouver une solution? Il faut comprendre le texte; si possible trouver des équivalents dans la langue d'arrivée pour tous les termes contenus dans le texte original. Le développement des langues de spécialité pour tous les domaines d'activité humaine force le traducteur à connaître ou à étudier la langue de spécialité qu'emploierait l'expert du domaine dont traite le texte à traduire dans sa propre langue, c'est-à-dire dans celle vers laquelle il traduit. Il doit donc pouvoir se documenter et puisqu'il est invariablement pressé à cause du délai assez rigoureux qui lui est imparti, il doit pouvoir disposer de moyens de recherche ultra-rapides.

Lorsqu'en 1960 l'idée m'est venue de créer un tel outil sous la forme d'un dictionnaire automatique, j'ai eu la chance de rencontrer madame Lydia Hirschberg, à l'époque directeur de l'Institut de linguistique automatique de l'Université libre de Bruxelles. Dans sa capacité d'expert en applications informatiques alphanumériques, elle a été d'un appui inestimable pour la conduite des travaux.

Le présent article n'est ni l'endroit, ni ne doit être un prétexte de décrire une fois encore en détail le cheminement et tous les aspects de la réalisation de notre projet, connu sous le nom de DICAUTOM ${ }^{1}$. Ici, il suffira de faire mention de ses grandes lignes.

Partant des prémisses qu'un mot ou un terme a souvent plus qu'une seule signification et que celle-ci dépend du contexte où il se trouve, il fut décidé de ne point entrer en mémoire électronique des simples mots, mais des locutions. Aussi voulions-nous éviter d'imiter les dictionnaires techniques bi- ou multilingues qui ne contiennent que des listes de mots dans une langue et en regard dans la ou les langues utilisées fréquemment, plusieurs mots sans aucune indication de leur emploi. La recherche d'un terme dans un contexte était rendue possible en consultant la mémoire moyennant une phrase qui comprenait le terme recherché. Cette phrase était extraite du texte à traduire. Le programme informatique permettait à l'ordinateur de comparer la phrase en question avec toutes les locutions en mémoire grâce à l'analyse linguistique qui s'y trouvait également pour chacune des langues du dictionnaire. Si l'équivalent exact ne se trouvait pas en mémoire, la réponse comprenait la ou les phrases présentant le plus grand nombre de mots contenus dans la phrase question ainsi que la traduction dans la langue demandée. Cette réponse fournissait au traducteur l'occasion de juger lequel des contextes proposés pré- 
sentait la plus grande ressemblance avec le texte original. En cas de doute, il pouvait consulter les documents dont les références étaient également fournies par l'ordinateur.

Le plus grand problème était évidemment celui de l'emmagasinage des termes. Une fois que le domaine était bien délimité, il fallait pour toutes les langues rassembler des textes valables et choisir ceux qui semblaient représenter le plus d'intérêt du point de vue de la langue de spécialité. Ensuite, il fallait pour chacun des termes sélectionnés décider de la longueur du contexte à y assortir. On avait d'abord pensé qu'en introduisant le texte complet en mémoire pour en tirer des listings KWIC, le tri des mots clés et de leurs contextes serait plus facile et plus rapide. Dans la pratique cette méthode s'avérait plus fastidieuse et plus lente que celle qui consistait à faire le choix en soulignant les termes et leurs contextes dans le texte. Une autre difficulté était de trouver les équivalents dans les autres langues, afin de pouvoir introduire en mémoire le langage réellement employé par l'expert du domaine lorsqu'il s'exprime dans sa propre langue et non pas une traduction littérale. Pour y arriver il fallait donc comparer des textes originaux traitant du même thème rédigés dans les différentes langues visées.

L'option prise d'introduire des mots dans un contexte nécessitait un travail de codage assez considérable. Lorsque les contextes adéquats faisaient défaut on décidait de les remplacer par la définition du terme de préférence en toutes les langues. Pour la consultation de DICAUTOM, il aurait fallu dès le début pouvoir disposer de plusieurs écrans et imprimantes, c'est-à-dire au moins un de chaque dans chacune des sections linguistiques du service de traduction. En raisons de difficultés budgétaires, ce désir ne pouvait se réaliser. Nous espérions contourner cette difficulté en proposant que les traducteurs reçoivent les textes à traduire en deux exemplaires. Ils souligneraient sur un des deux les termes à rechercher. Cet exemplaire serait adressé au Bureau de terminologie qui consulterait le dictionnaire automatique et leur retournerait le texte où figurait les soulignements accompagné d'une liste où figureraient les réponses obtenues en ordre chronologique. Cette solution ne pouvait pas être adoptée à cause de la pénurie de personnel tant au service informatique qu'au Bureau de terminologie. D'ailleurs les traducteurs eux-mêmes manifestèrent des réserves puisqu'ils craignèrent perdre toute possibilité de recherches terminologiques personnelles en leur imposant des traductions pour ainsi dire préfabriquées.

En attendant, puisque DICAUTOM ne contenait pas encore une collection de termes suffisamment grande et variée, il fallait se résigner à n'utiliser DICAUTOM que pour la confection de glossaires phraséologiques multilingues à l'occasion d'événements tels les Congrès de l'acier'2.

En 1965, la fusion des trois exécutifs des Communautés européennes causait par la disparition de la Haute Autorité l'arrêt complet des travaux de développement des programmes informatiques. Toutefois, l'analyse terminologique de textes par les terminologues continuait afin de permettre de constituer un stock grandissant de termes en vue de son entrée en mémoire lorsque les programmes pouvaient être repris. Malgré le sens de frustration dont souffraient tous ceux qui avaient participé avec tant d'enthousiasme à la création de ce dictionnaire automatique leurs efforts prouvaient ne pas avoir été vains, lorsque les travaux ont pu reprendre dans toute leur ampleur en 1973. L'intérêt manifesté par l'Université de Montréal d'abord et par le Bureau des traductions du Secrétariat d'État à Ottawa ensuite, a inconstestablement contribué à convaincre l'administration dont dépendait le Service de traduction et le Bureau de terminologie de rendre possible le redémarrage.

Ce nouveau départ a également été inspiré par l'appui efficace de la direction générale qui à la Commission, l'exécutif unique après la fusion, est responsable pour tout ce qui concerne l'information et les bases de données ainsi que leurs réseaux d'échanges. 
Madame Hirschberg ayant quitté l'Université libre de Bruxelles pour assumer d'autres fonctions, les programmes ont dû être remaniés et complétés par les informaticiens de la Commission dans la lumière de l'expérience acquise. Afin de bien faire ressortir qu'il s'agit d'une banque de terminologie au service de tous les traducteurs européens un nouveau titre EURODICAUTOM a été choisi. Cette banque de terminologie est accessible à tous les intéressés sur EURONET, le réseau de bases de données géré par la Commission des Communautés européennes.

Afin de rapidement augmenter le stock terminologique de la banque, des collections de termes sans contexte mises à la disposition par Montréal, Ottawa et plusieurs autres organisations qui s'occupent activement de terminologie, ont été mises en mémoire. Les responsables ont aussi décidé d'opter pour un mode de consultation basé sur des termes isolés au lieu de sur des locutions (termes entourés d'un contexte). Pour remédier à l'absence de contextes, les termes ont été complétés par des définitions, du moins pour les langues de grande diffusion.

A la suite de la restructuration des services de traduction dont la Commission dispose à Bruxelles et à Luxembourg, un service unique de terminologie et des applications informatiques a vu le jour, sous la direction de Jacques Gœtschalckx. Cette nouvelle structure a provoqué une collaboration étroite entre tous les terminologues au service de la Commission. Le résultat en est une accélération considérable dans l'introduction de nouveaux termes ainsi que de l'épuration du stock existant. Ainsi, les lacunes pour les langues de moindre diffusion sont également comblées avec plus de dextérité.

L'élargissement de la Communauté par l'adhésion de la Grèce, au début de 1981, et de l'Espagne et du Portugal au 1er janvier 1986, a causé l'augmentation du nombre des langues officielles d'abord de six à sept et puis à neuf. EURODICAUTOM a pu faire face à ce développement grâce à la collaboration internationale grandissante dans le domaine de la terminologie et de la documentation.

Après de longues années d'initiation et aidé par l'apparition de l'ordinateur dans de nombreux secteurs d'activité humaine, les traducteurs acceptent aujourd'hui plus facilement de consulter une banque de terminologie et en reconnaissent l'utilité. Les efforts et les peines ainsi que les angoisses des temps héroïques des essais n'ont donc pas été vains. Et tous ceux qui y ont collaboré peuvent en être fiers.

Et l'avenir? Qu'est-ce qu'il réservera aux banques de terminologie? Nous pensons et nous le souhaitons que l'avenir produira une collaboration et une intégration toujours grandissantes entre toutes les banques de terminologie dans le monde. L'interchangeabilité des systèmes devra être intensifiée afin d'éviter les doubles emplois dans toute la mesure du possible. Nous nous attendons aussi à une simplification des modes de consultation, de sorte que l'utilisateur n'aura plus besoin de taper ses questions sur un clavier, mais pourra les poser oralement au moyen d'un micro.

Il devra également être possible d'introduire les textes à analyser directement en mémoire sans passer par l'encodeuse et de choisir sur l'écran les termes destinés à la banque de terminologie en les soulignant avec un crayon spécial.

En l'an 2000, le contenu des banques de terminologie sera devenu si volumineux qu'il sera indispensable d'en faire des extraits qui seront introduits dans des minimémoires qui fonctionneront comme les machines à calculer de poche dont tout le monde se sert de nos jours.

Mais toutes ces banques de terminologie, gigantesques ou miniaturisées, ne serviront pas à grand chose, si les hommes ne réalisent pas que pour une bonne compréhension des idées et des inventions il faudra s'exprimer dans un langage clair en utilisant une terminologie adéquate et en évitant d'employer des termes peu usuels ou inexacts. C'est seulement lorsque tout le monde est enfin convaincu de l'importance que revêt le 
bon usage de la langue et de la terminologie que tous ces outils sophistiqués pourront pleinement donner satisfaction.

\section{Notes}

1. Cf. Actes de la $2^{\mathrm{e}}$ Conférence internationale sur le traitement automatique des langues, Grenoble, 23-25 août 1967 et Actes du Colloque international de linguistique et de traduction, Montréal, Canada, 30 septcmbre-3 octobre 1970, publiés dans META.

2. $1960:$ l'Acier dans les charbonnages; 1965 : l'Acier dans le bâtiment ; 1967 : l'Acier dans l'agriculture ; 1969 : l'Acier dans l'industrie chimique. 